Cell Research (2002); 12(1):19-32

http://www.cell-research.com

REVIEW

\title{
Structure and function of aggrecan
}

\author{
Chris KIANI ${ }^{1}$, Liwen CHEN ${ }^{1}$, YAo Jiong WU ${ }^{1}$, Albert J YEE², Burton B YANG ${ }^{1, *}$ \\ Sunnybrook and Women's College Health Sciences Centre and ${ }^{1}$ Department of Laboratory Medicine and \\ Pathobiology, ${ }^{2}$ Department of Surgeny, Faculty of Medicine, University of Toronto, Canada
}

\begin{abstract}
Aggrecan is the major proteoglycan in the articular cartilage. This molecule is important in the proper functioning of articular cartilage because it provides a hydrated gel structure (via its interaction with hyaluronan and link protein) that endows the cartilage with load-bearing properties. It is also crucial in chondroskeletal morphogenesis during development. Aggrecan is a multimodular molecule expressed by chondrocytes. Its core protein is composed of three globular domains (G1, G2, and G3) and a large extended region (CS) between G2 and G3 for glycosaminoglycan chain attachment. G1 comprises the amino terminus of the core protein. This domain has the same structural motif as link protein. Functionally, the G1 domain interacts with hyaluronan acid and link protein, forming stable ternary complexes in the extracellular matrix. G2 is homologous to the tandem repeats of G1 and of link protein and is involved in product processing. G3 makes up the carboxyl terminus of the core protein. It enhances glycosaminoglycan modification and product secretion. Aggrecan plays an important role in mediating chondrocyte-chondrocyte and chondrocyte-matrix interactions through its ability to bind hyaluronan.
\end{abstract}

Key words: Proteoglycan, chondroitin sulfate, glycosaminoglycan, G1 domain, G3 domain.

\section{INTRODUCTION}

Degenerative joint disease is a leading source of morbidity resulting in significant social and economic impact. One to $5 \%$ of the population under the age of 45 and $15-85 \%$ of older individuals suffer from some form of degenerative joint disease, mainly

\footnotetext{
* Correspondence to: Burton B. Yang, Research Building, Sunnybrook and Women' s College Health Sciences Centre, 2075 Bayview Avenue, Toronto M4N 3M5 Canada. Tel: (416) 480-5874; Fax: (416) 480-5737; E-mail: Burton.Yang@swchsc.on.ca

The abbreviations used are: ECM, extracellular matrix; CS, chondroitin sulfate (chain attachment sequence); KS, keratan sulfate (chain attachment sequence); GAG; glucosaminoglycan; HA, hyaluronan; G1, the globular domain in the N-terminus of aggrecan; G2, second globular domain of aggrecan; G3, the globular domain in the carboxyl terminus of aggrecan or selectin-like domain; IGD, inter-globular domain; PTR, proteoglycan tandem repeat; EGF, epidermal growth factorlike motif; CRD, carbohydrate recognition domain; CBP, complement binding protein.
}

osteoarthritis. Osteoarthritis is characterized by the slow progressive deterioration of articular cartilage [1], [2]. Current therapeutic regimens address mainly pain but not degeneration. A better understanding of the distinct micro-environment of articular cartilage and the complex interactions that exist between cell and surrounding extracellular matrix (ECM) is evolving so that strategies can be directed towards altering the natural history of degenerative joint disease.

The gliding surfaces of synovial joints are covered by articular cartilage. Articular cartilage is composed of hyaline cartilage, which provides a thin, smooth, stiff and wear-resistant layer that provides a low friction weight bearing joint surface that allows our joints to move smoothly and without pain. The destruction of articular cartilage that occurs with degenerative joint diseases that leads to joint dys- 
function and pain.

Cartilage is composed of the cells named chondrocytes and the ECM produced by these cells. The biochemical properties of cartilage and the physical function of joints are critically dependent on the integrity of the matrix. The ECM molecules in cartilage include proteoglycans, hyaluronan (also called hyaluronic acid or HA), type II collagen, glycoproteins and various mixtures of elastic fibers. Proteoglycans are a family of glycoconjugates with a central core protein to which one or more glycosaminoglycan (GAG) side chains are covalently linked post-translationally [3]. In addition, most of the proteoglycans exist as aggregates[4] formed by the non-covalent association of proteoglycan with HA and link protein[5-7]. Among the cartilage proteoglycans, the most crucial to the proper functioning of articular cartilage is aggrecan, one of the large aggregating chondroitin sulfate proteoglycans. Aggrecan is a multimodular molecule and here, we will review the structures and functions of its modules.

\section{Aggrecan and its environment}

Cartilage contains up to $10 \%$ proteoglycan consisting of mainly the large aggregating chondroitin sulfate proteoglycan aggrecan. The aggrecan family includes other important members such as versican, also named PG-M, neurocan, brevican and the cell surface HA receptor CD44[8-11]. They are modular proteoglycans containing combinations of structural motifs, such as epidermal growth factor (EGF)-like domains, carbohydrate recognition domains (CRD), complement binding protein (CBP)-like domains, immunoglobulin folds and proteoglycan tandem repeats[12]. At high concentrations, proteoglycans create a large osmotic swelling pressure and draw water into the tissue (Fig 1). This occurs because all of the negatively charged anionic groups on the GAG chains of aggrecan carry with them mobile counter ions such as $\mathrm{Na}^{+}$. This creates a large difference in ion concentration between the cartilage and surrounding tissue and an imbalance amongst the freely diffusible anions and cations. Water is drawn into cartilage because of this osmotic imbalance and because aggrecan is too large and immobile to redistribute itself. The addition of water causes aggrecanrich matrix network to swell and expand. This wa- ter-swollen matrix is critical to the biomechanical properties of cartilage. Another feature of the composite collagen/aggrecan organization is also important. Not only is aggrecan greatly restricted in its ability to move within the matrix, but also the collagen/aggrecan network is stiff and resistant to deformation. Aggrecan also offers great resistance to any fluid flow and redistribution of water. Thus, cartilage is referred to as a visco-elastic tissue in that it behaves like the stiff elastic polymer resistant to sudden impact loading, yet shows some slow inelastic deformation with sustained loads[13].

\section{Aggrecan structure and functions}

In cartilage, aggrecan is found in huge multimolecular aggregates, comprised of numerous monomers non-covalently bound to HA. The small glycoprotein link protein, which is homologous to the Nterminus of aggrecan, helps to stabilize aggregate formation (Fig 2). The GAG side chains contribute to the formation of the large mass aggregate. Almost $90 \%$ of aggrecan mass is comprised of substituted GAG chains which are mostly chondroitin sulfate chains, but also include keratan sulfate chains with $\mathrm{N}$ - and O-linked oligosaccharides.

Aggrecan has three globular domains (G1, G2 and G3) and three extended domains (IGD, KS and CS) as shown in Fig 3. Following the signal peptide is the N-terminal G1 domain. An inter-globular domain (IGD) connects G1 and G2 domains. Situated between the G2 domain and G3 domain is a large sequence modified by KS CS side chains. Each aggrecan contains $\sim 100$ chondroitin sulfate chains, which are typically $\sim 20 \mathrm{kDa}$ each. There are fewer keratan sulfate chains (up to 60) and they are usually of smaller size (5-15 kDa). Aggrecan also contains a variable number of $\mathrm{O}$ - and $\mathrm{N}$-linked oligosaccharides. The O-linked oligosaccharides have a linkage to protein similar to that of keratan sulfate. During biosynthesis, some O-linked oligosaccharides are extended and sulfated to form keratan sulfate chains.

All three of the globular domains of the aggrecan protein contain sequences that are highly conserved amongst aggrecan from different species, while the extended domains are less conserved. The mouse aggrecan gene spans at least $61 \mathrm{~kb}$ and contains 18 exons. Exon 1 encodes 5'-untranslated sequence and 
exon 2 contains a translation start codon. The coding sequence is $6545 \mathrm{bp}$ for a 2132 -amino-acid protein with calculated molecular weight of $259,131 \mathrm{Da}$, including an 18-amino acid signal peptide. There is a strong correlation between structural domains and exons. Notably, the chondroitin sulfate domain consisting of 1161 amino acids is encoded by a single exon of $3.6 \mathrm{~kb}$.

Aggrecan G1 domain is in fact similar to link protein and other members of this proteoglycan family, in terms of its structural domains and subdomains. There are also stretches of sequences similar to the promoter region of both the type II collagen and link protein genes. These sequences may be important for cartilage gene expression[14]. The amino acid sequences of human aggrecan and rat aggrecan are about $75 \%$ identical. The human sequence contains two regions of highly conserved repeats not found in rat aggrecan: 11 repeats of a hexameric sequence in the keratan sulfate attachment domain, E-E-P-(S/ F)-P-S; and a 19-amino acid sequence reiterated 19 times, in the CS-1 portion of the serine- glycinecontaining region. There are at least three forms of aggrecan transcripts, generated by alternative exon usage, with or without EGF-like motifs[15].

As a structural proteoglycan, aggrecan appears to be important in mediating chondrocyte-chondrocyte and chondrocyte-matrix interactions. Co-expression of a mini-aggrecan and link protein stabilizes cell-substratum interaction[16]. Addition of exogenous gene products into fibroblast cell lines and chondrocyte culture had the same effect as expression of the genes. The addition of exogenous HA to the growth medium, or treatment of cells with HA,

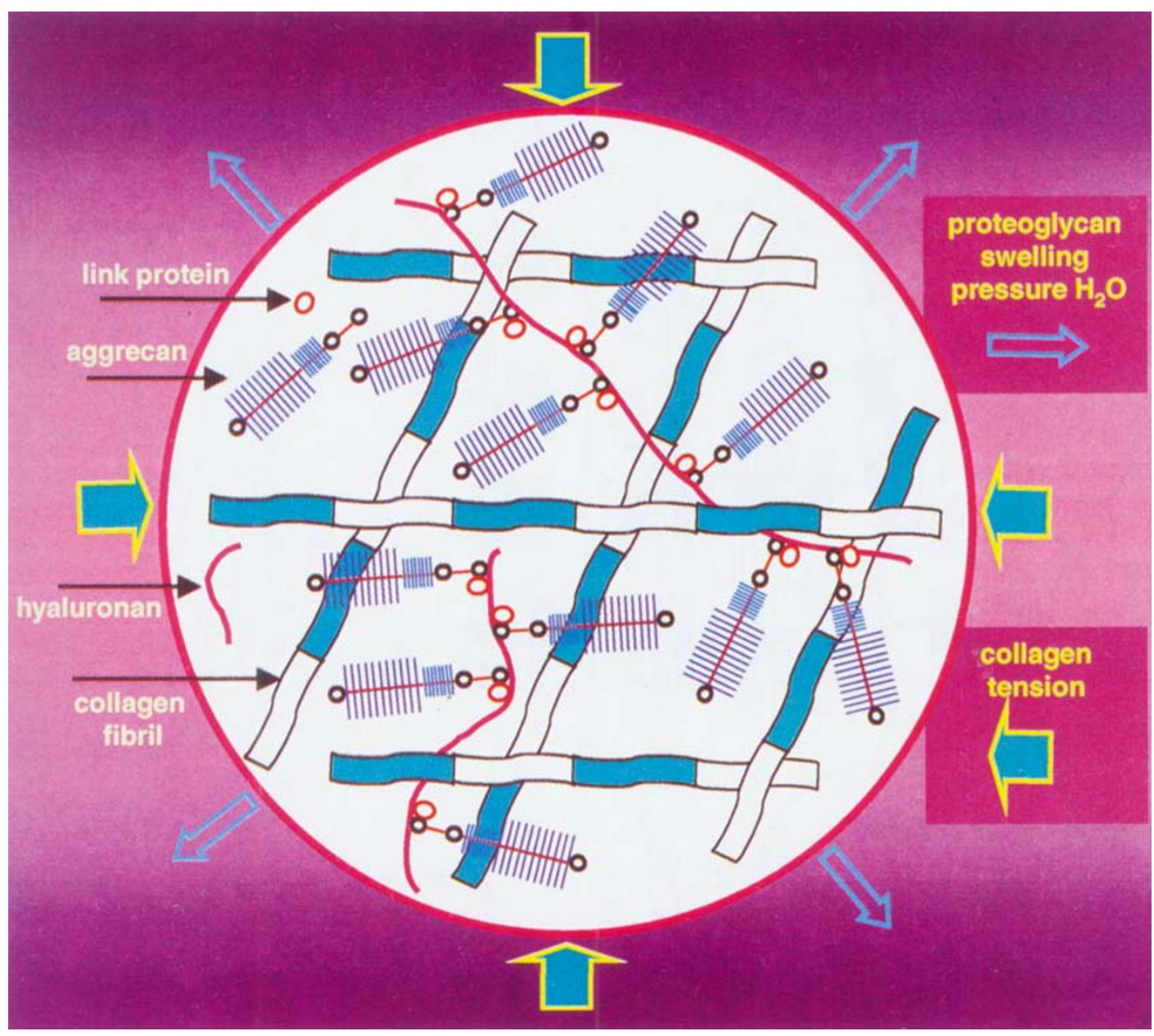

Fig 1. The combined properties of collagens and aggrecan in articular cartilage 


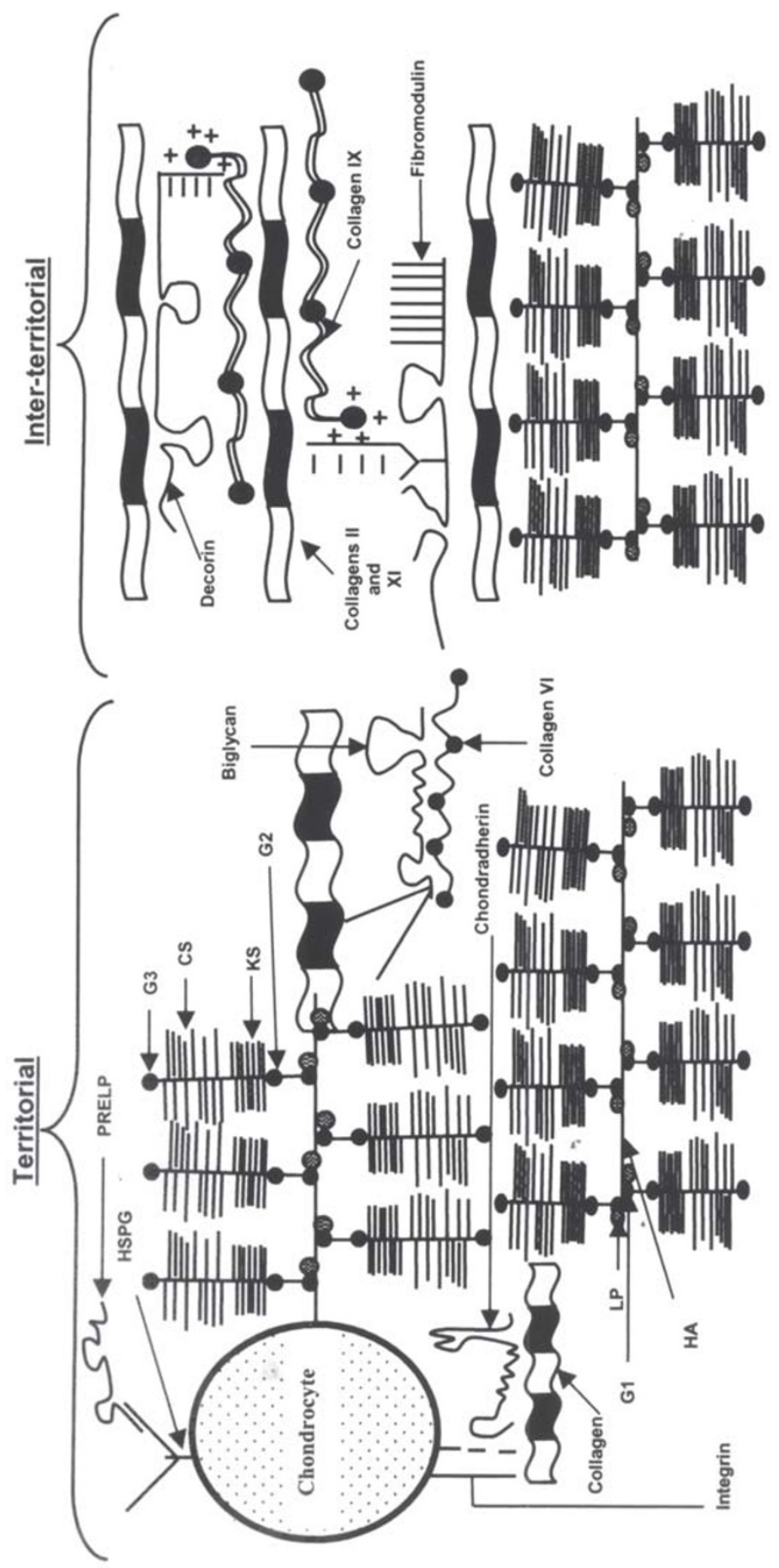

造离

द्व 0 पू

की

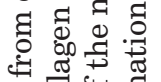

녕 न्ठ है

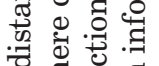

起专

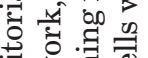

용

ఖ

要 要

'ृ

की

a

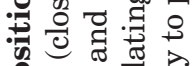

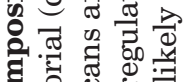

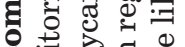

० $\Rightarrow$

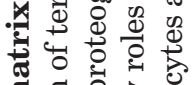

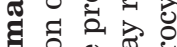

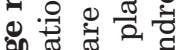

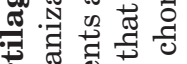

हैं

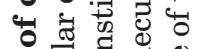

‡

刍家总

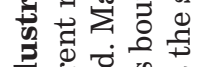

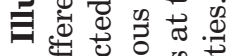

ง

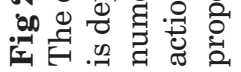


also decreased cell adhesion, indicating that $\mathrm{HA}$ also plays a role in the cell-substratum adhesion. The presence of aggrecan seems to increase the amount of link protein on the cell surface. Chondrocytes expressing high concentrations of aggrecan and link protein were maintained within a matrix network and were able to survive in suspended culture. Imbalances in aggrecan or link protein concentrations, or degradation of HA, disrupted the network and caused the chondrocytes to aggregate or adhere to the plates[16],[17]. Digestion of HA also induces chondrocyte aggregation. The effect of HA on cell aggregation and cell attachment seems to predominate over aggrecan, type II collagen and link protein[17]. Another member of the large aggregating chondroitin sulfate proteoglycans, versican, also plays a role in mediating cell adhesion and migration[18]. As versican is expressed in a variety of tissues, its functions are also diverse. Versican has been shown to be able to promote the growth of NIH3T3 fibroblasts and chicken chondrocytes[19]. It also inhibits mesenchymal chondrogenesis of chicken limb buds
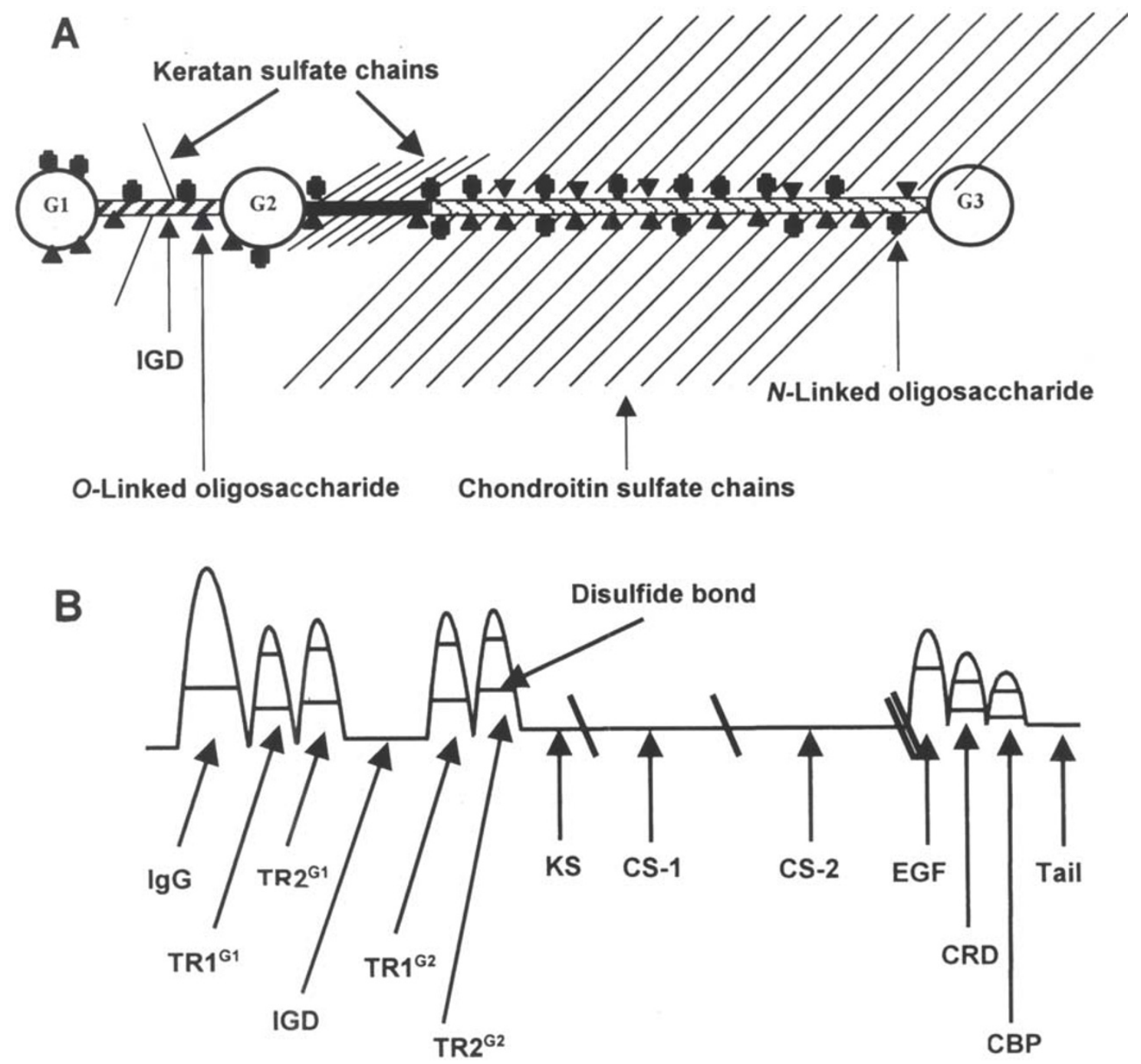

Fig 3. Aggrecan structure

(A) Globular protein and attached GAG chain structure. (B) Protein domain structure. Folded modules: IgG, immunoglobulin fold: TR, tandem repeats: EGF, epidermal growth factor-like module: CRD, carbohydrate recognition domain: CBP, complement binding protein-like module. Extended domains: IGD, interglobular domain: KS, keratan sulfate attachment domain: CS-1 and CS-2, chondroitin sulfate attachment domains. 
[20]. Expression of a mini-versican construct alters chondrocyte morphology[21]. However, it is not clear whether aggrecan has similar effects on cell activities.

\section{THE G1 DOMAIN}

G1 domain is encoded by exons $3,4,5$ and 6 . This domain is comprised of three modules: an immunoglobulin fold, and two copies of a HA-binding motif, or link module, also referred to as the proteoglycan tandem repeat (PTR) (Fig 3). The immunoglobulin fold motif of aggrecan G1 corresponds to the hypervariable region of the immunoglobulins and is about 100 amino acids long. This region is predicted to fold into two $b$ sheets in a sandwich conformation (parallel orientation) stabilized by a conserved disulfide bond (Fig 3). This motif is encoded by a single exon. Almost all of the proteins that contain immunoglobulin-related structures are cell surface molecules involved in cell recognition, cell adhesion or immune reaction. Their roles in cell recognition are corroborated by the fact that many of these molecules interact with other members of the immunoglobulin superfamily, and indeed the interaction between aggrecan G1 domain and link protein is mediated via the immunoglobulin fold[22]. The PTR is present in duplicate in all members of the HA-binding family of proteoglycans including versican, neurocan and brevican. However, it is present as a single copy in the cell surface HA-binding receptor, CD44, and in TSG, a secreted matrix protein whose synthesis is induced by inflammatory cytokines. The PTR of the G1 domain are comprised of two cysteine-rich motifs which are able to form disulfide bonds (four cysteine residues or two disulfide bonds per tandem). The tandem repeats, and particularly the disulfide bonds, are involved in aggrecan' $\mathrm{s}$ interaction with HA, forming the large complexes (Fig 3). These tandem repeats do not bind to HA under reducing conditions[23].

In addition to its interaction with HA, the G1 domain also interacts with link protein, and thus forms the "glue" holding together the ternary complex in the matrix. The ternary complex plays a critical role in maintaining a stable matrix network in cartilage. Due to its binding activities, the G1 domain mediates interactions between chondrocyte and the matrix network. Overexpression of the G1 do- main has been shown to reduce the adhesion of chicken chondrocytes[24]. The reduced cell adhesion enhances cell apoptosis[25]. The G1 domain of versican also reduces NIH3T3 fibroblast adhesion [26]. Recently, it was observed that the G1 domain of aggrecan regulates product processing[27]. The same effect was also observed for versican G1 domain[28]. In both cases, the G1 domains inhibit GAG modification and product secretion. These functions appear to play a key role as a checkpoint for proteoglycan quality control.

\section{The inter-globular domain (IGD)}

The short extended region separating the G1 and G2 domains of aggrecan is known as the interglobular domain (IGD). This domain has a rod-shaped structure in which contains proteolytic cleavage sites susceptible to a variety of proteinases such as matrix metalloproteinases (MMPs), serine proteinases such as plasmin and leukocyte elastase, and acid proteinases such as cathepsin B (cysteine protease) (Fig 4) [1],[29],[30]. The IGD domain is encoded by exon 7 of aggrecan. It is unique to the aggrecan molecule since other members of the aggrecan family lack this region. X-ray diffraction, NMR (nuclear magnetic resonance imaging) and rotary shadowing electron microscopic analyses have determined that the IGD is of constant length $(25 \mathrm{~nm})$ and relatively stiff and inflexible[31],[32]. This apparent stiffness of the IGD may be due to the density of keratan sulfate substitution[33],[34], which seems to be greater in the IGD region than the adjoining globular domain (G2). The functional, rather than structural, properties of IGD make it interesting. The IGD domain is the site of proteolytic attack on aggrecan during pathological cartilage degradation. Cleavage of aggrecan molecules in the IGD region near its G1 domain results in rapid loss of the whole GAG attachment region. As such, IGD appears to be involved in the physiological turnover of aggrecan.

Key observations-that fragments harboring a G1 domain bearing a VDIPEN-341 neoepitope (rather than a NITEGE-373 neoepitope) are present in human synovial fluids[35] and appear rapidly in rabbit knee joint fluid after an intra-articular injection of MMP3[36]-suggest that there is further processing of the HA-G1 complex after the IGD has been cleaved off by aggrecanase and has diffused out of 
the matrix. Recent work on the extent of the involvement of aggrecanase and MMPs in the cleavage of aggrecan at the IGD domain indicates that aggrecanase cleavage of aggrecan by far exceeds cleavage by MMPs under patho-physiological conditions[37].

Two major cleavage sites have been identified in the IGD domain: one occurs between residues Asn 341 and Phe 342 and the other is located between residues Glu 373 and Ala 374. The first major cleavage site generates a G1-containing fragment with the C-terminus neoepitope VDIPEN-341 and a larger GAG-bearing fragment with the $\mathrm{N}$-terminus neoepitope 342-FFGVGGE[34], [38-40]. The results of many studies support the contention that MMPs are involved in the breakdown of aggrecan molecules in vivo. First, cartilage and synovial fluid from patients with osteoarthritis and other inflammatory arthritis types contain enhanced levels of MMPs. Second, retinoic acid and pro-inflammatory cytokines (IL-1 and TNF- $\alpha$ ) are known to upregulate the expression of several MMPs, and this promotes aggrecan loss from cartilage explants[41-43]. This loss of aggrecan can be prevented by adding specific inhibitors of MMPs to the conditioned medium[44], [45]. Third, the N- and C-terminal neoepitopes generated by MMPs are present in both cartilage and synovial fluid. Finally, it has been demonstrated that the concentration of the VDIPEN-341 neoepitope in normal human articular cartilage increases up to 25 years of age and then reaches an apparent steady state (representing 15-20\% of the G1-containing molecules residing within the matrix)[46]. This suggests that MMP-generated G1 fragments might account, at least in part, for the growth- and maturation-related increase in aggrecan domains in the cartilage.

Studies on the aggrecan fragments extracted from articular cartilage or synovial fluid suggested the presence of a proteinase that cleaves a specific site in IGD, which was named "aggrecanase” [47]. Aggrecanase1 is a member of the ADAMTS (a disintegrin and metalloproteinase with thrombospondin motifs) protein family that cleaves aggrecan at the Glu373-Ala374 bond[48]. This cleavage produces a large GAG-rich aggrecan fragment with the $\mathrm{N}$-terminus neoepitope 374-ARGSVI, and a G1 fragment with the C-terminus neoepitope NITEGE-373 (Fig 4). Aggrecanase- mediated cleavage occurs both in vivo and in vitro: the N-terminus neoepitope 374-ARGSVI has been found in the medium of cartilage explant cultures [49] as well as in high density aggrecan fragments recovered from human synovial fluids[47],[50],[51]. It is believed that MMPs and aggrecanase are both involved in the turnover of aggrecan molecules in normal and diseased cartilage. This is supported by the finding that the MMP-generated G1 fragment terminating in VDIPEN-341 and aggrecanase- generated G1 fragments terminating in NITEGE-373 are both detected in cartilage from joints with osteoarthritis and rheumatoid arthritis[40]. The generation and/or turnover of these specific aggrecan fragments is not necessarily co-ordinated, since both the NITEGE-373 and VDIPEN-341 neoepitopes can be non-coincident within a single joint[38]. Turnover of aggrecan in cultured rat chondrosarcoma cells and primary bovine chondrocytes can be mediated exclusively by aggrecanase[52].

It has been reported that aggrecanase also cleaves off aggrecan at multiple sites in the CS-attachment domain[2],[53],[54] generating GAG-rich fragments, which are lost relatively rapidly from the cartilage matrix. These fragments diffuse into the synovial fluid, where they can be quantified by chemical assays of their sulfated GAG chains[55] or by immunoassays capable of measuring specific protein or carbohydrate epitopes[53], [54]. Recently, it has been shown that cathepsin B (a cysteine protease with both endopeptidase and carboxypeptidase activities) is able to cleave aggrecan. Cleavage occurs at Asn341-Phe342, to yield the neoepitopes VDIPEN and FFGVGG[30].

\section{The G2 domain}

This domain is encoded by exons 8,9 and 10 of the aggrecan gene and is the second globular domain from the $\mathrm{N}$-terminus. It consists of two proteoglycan tandem repeats, which are very similar to those of the G1 domain. Aggrecan is the only molecule of the aggregating chondroitin sulfate proteoglycan family to contain this domain. It is noteworthy that aggrecan is also the only member of this family that is heavily glycosylated by $\sim 100$ chondroitin sulfate and up to 60 keratan sulfate chains. There may be a direct or indirect role for the G2 domain in GAG chain attachment. 
The G2 domain shows an approximately $67 \%$ amino acid sequence similarity to that of the tandem repeats in the G1 domain. Whereas the tandem repeats have been shown to be the major HA-binding element in link protein and the G1 domain, the G2 domain, either purified from cartilage after proteolytic digestion[56] or the recombinant soluble G2 protein [57], shows no HA-binding function whatsoever.

The G2 domain has also been shown to contain keratan sulfate side chains[58]. While it is not clear where the KS is attached, it may also significantly interfere with the ability of the G2 domain to interact with HA. The locations of cysteine residues essential for maintaining the three-dimensional structure of the G2 domain, and the locations of asparagine residues required for attaching carbohydrate in an N-linked fashion, are well conserved. The G2 domain is not able to bind to link protein, other aggregating proteoglycan monomers, collagen or other major components of the dissociate extract of cartilage[56]. Nevertheless, we have recently shown that the G2 domain inhibits product secretion[27]. The product of a recombinant G2 construct is not secreted, even when linked to a signal peptide. Secretion of other recombinant products containing the G2 domain is either inhibited or retarded. This is so far the only function assigned to the G2 domain, and may be associated with product quality control in order to produce a mature functional aggrecan molecule.

\section{The KS domain}

Following the G2 domain is the keratan sulfate (KS) domain, which is encoded by exon 11. There are about $30 \mathrm{KS}$ chains attached to the mature aggrecan molecule. The amino acid sequence of this domain varies among different species. The potential consensus sequence for the attachment of $\mathrm{KS}$ chains in the human is E-(E/K)P-F-P-S or E-E-P-(S/ F)-P-S[15]. Mice and rats lack these sequences and also lack keratan sulfate chains in their articular aggrecan. In both bovine[59] and human aggrecan [15], the amino acid sequence of this region, revealed by cDNA sequence analysis, contains a striking repeating sequence. The sequence is a hexamer, which contains a serine residue. It has been suggested that proline-serine and proline-threonine repeats in this region are good candidates for substitution by KS chains. The human aggrecan cDNA encodes an additional 66-residue-sequence consisting of a highly conserved hexamer peptide motif repeated 11 times consecutively. This structure is consistent with the fact that human aggrecan is more heavily glycosylated in this region than is rat aggrecan. Both bovine and rat aggrecan also contain this sequence, but contain fewer repeats[15].

The KS region is not the only site of KS attachment in the protein core. KS is also distributed elsewhere, primarily in the CS region. Some of the KS chains are O-linked to threonine[60]. It is possible that the repeating Thr-Thr-Ala-Pro sequence in the N-terminal end of the CS region represents this attachment site. KS chains from bovine aggrecan have a molecular weight of approximately of $10 \mathrm{kDa}$ [61]. The KS chains vary in structure depending on tissue source. KS from load-bearing tissues (articular cartilage and intervertebral disks) contain 1-3 fucose residues and 2-6 N-acetyl neuraminic acid residues, which are absent from non-load-bearing tissues (tracheal and nasal cartilage)[62]. The KScontaining peptide isolated from chick aggrecan does not exhibit significant similarity to the human or bovine KS domain and may represent a further variation in this domain[63]. It has been reported that the concentration of keratan sulfate in human cartilage (especially menisci) increases with age as does the concentration of the 6-sulfated disaccharide of chondroitin sulfate does[64].

The function of this domain is not very clear. It may be involved in tissue distribution of aggrecan. The keratan sulfate chains may contribute to tissue development. Recently, we observed that the KS domain plays a role in product processing[27]. Secretion of some recombinant products was enhanced in the presence of a KS domain. Last, but not least, the keratan sulfate chains in this domain hold water within aggrecan molecule, and thus enhance the load-bearing capability of aggrecan in cartilage.

\section{The CS domain}

The chondroitin sulfate (CS) domain is the largest domain of aggrecan and is decorated by approximately 100 chains of chondroitin sulfate. This domain is encoded by a single exon, exon 12 , with a size of $\sim 3.5 \mathrm{~kb}$. The CS domain consists of approximately 120 Ser-Gly dipeptide repeats. The dipeptides are frequently separated by an acidic residue 

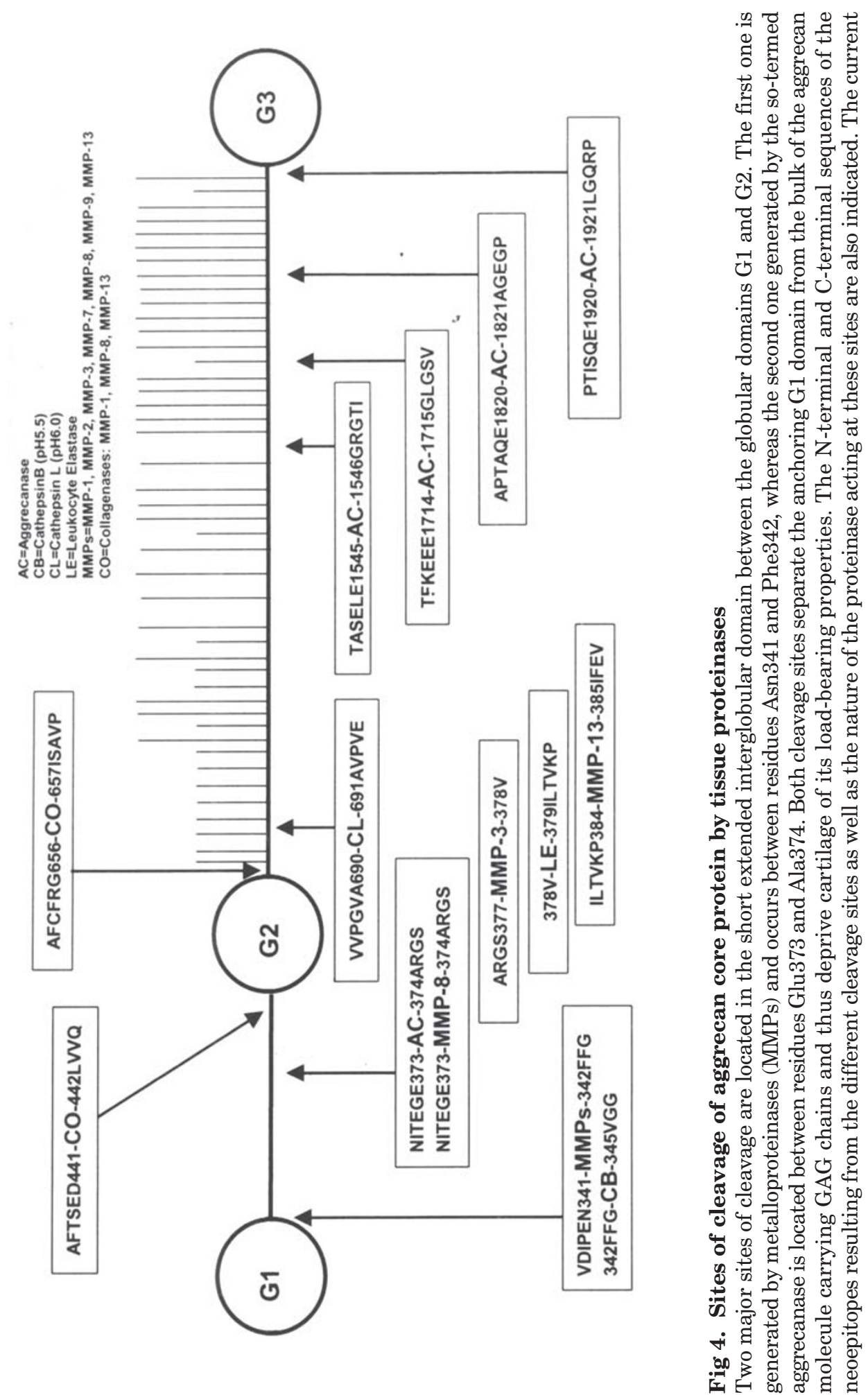
and a hydrophobic residue[63]. The recognition sequences for the attachment of CS chains have been proposed to be S-G-X-G(65) or (D/E)-X-S-G(63). In addition to the primary sequence, molecular chaperone surveillance mechanisms and localization of enzymes for post-translational modifications may also be necessary for the recognition.

In proteoglycans, many, but not all, Ser-Gly dipeptides are substituted, often in regions containing flanking acidic residues. A comparison of CS substitution sites in three proteoglycans-decorin[66], [67], rat yolk sac tumor proteoglycan [68], [69] and the invariant chain of human class II MHC complex molecules[70]- has identified the tetrapeptide, -SerGly- Xaa-Gly- (where X can be any amino acid) as a good substrate for xylosyltransferase[65]. For example, aggrecan appears to have a consensus sequence with a decapeptide repeat containing two Ser-Gly dipeptides separated from a second pair of Ser-Gly dipeptides[15],[71]. Another sequence, acidic-Gly-Ser-Gly-acidic, is prominent in human versican and has also been identified as the CS attachment site in the $\alpha 2$ chain of chicken type IX collagen [72]. Gly-Ser-Gly triplet repeats and GlySer pairs seem to be more common in chicken PGM than are Ser-Gly pairs[9].

The negatively-charged chondroitin sulfate chains in this domain account for the major function of aggrecan as a structural proteoglycan: its ability to hold large amount of water in the ECM. The waterholding property of the GAG chains may also play an important role in aggrecan processing. Indeed, we have recently shown that addition of GAG chains to the CS sequence play an important role in product secretion[27]. Without GAG modification, products of CS-containing constructs are not secreted. To allow sufficient modification of GAG chains, a G3 domain is essential.

\section{The G3 domain}

The G3 domain is a complex region produced by alternative splicing of exons during post-transcriptional processing. This domain consists of three modules: the EGF-like module, the CRD and the CBP module as well as a short tail. In humans, variable alternative splicing results in about one quarter of aggrecan molecules containing an EGF-1 and a small proportion containing EGF-2 or both modules[73]. In mice, rats, and dogs, EGF-1 is a part of an intron and is not translated. In chicken, alternative splicing gives rise to aggrecan molecules with only one, or in some cases none, of the EGF modules encoded by exon 14. The submodules of CRD and CBP in chicken are encoded by exons 14, 15, 16,17 and 18 respectively.

Functionally, the CRD module of aggrecan binds to fucose and galactose[74]. The recombinant C-terminal region (EGF-like module, C-type lectin-like module, and CBP module) of PG-M/versican can bind to heparin and heparan sulfate[75]. The lectin-like module of versican binds to tenascin- $R$ by protein-protein interactions[76]. Comparison of tenascin- $\mathrm{R}$ interactions among a family of the G3 domain-containing proteoglycans revealed not only a carbohydrate-protein interaction, but also a distinct protein-protein interaction[77]. The aggrecan G3 domain also appeared to be involved in the processing of this proteoglycan. This was initially highlighted by two autosomal recessive mutations in animals, nanomelia in chickens and cartilage matrix deficiency in mice.

In nanomelic aggrecan, a point mutation at position 4553 at the end of exon 12 encoding the CS domain converts the codon GAA (glutamate) to TAA giving rise to a stop codon at amino acid position 1513. This single mutation leads to a shortened core protein precursor with a calculated molecular weight of $158 \mathrm{kDa}$. The resulting phenotype, nanomelia, arises because the truncated core protein is neither processed nor secreted from the chondrocytes[78]. It is now known that, without the G3 domain, the truncated protein core of aggrecan cannot be modified by GAG, and the unmodified protein core cannot be secreted. As a result, the cartilage, becomes thinner, and the long bone. Homozygous nanomelic chickens die shortly after birth due to severe skeletal problems.

Cmd, cartilage matrix deficiency is caused by a single 7-bp deletion in exon 5 (which encodes the first tandem repeat loop of the G1 domain) of the aggrecan gene. This deletion causes a frameshift resulting in the introduction of a termination codon in exon 6[79]. The afflicted homozygous cmd animals die shortly after birth due to respiratory 
problems. The heterozygous animals die after 12-15 months, whereas wild type mice live for 2-2.5 years. Even though the cmd+/cmd- animals appear normal at birth, later in life they develop dwarfism[80]. There are multiple reports on the role of G3 modules in aggrecan secretion and post-translational modifications. One study in chicken suggests that aggrecan G3 exerts its functions mainly through its lectin-like modules encoded by exon 15[81].

Based on these animal models, it has been proposed that G3 may regulate the attachment of GAG chains and affect the secretion of aggrecan. This has later been demonstrated to be so in aggrecan[27] and versican[28]. It is believed that G3' sfunctions in product processing are important for product quality control through the ubiquitin-proteosome dependent degradation pathway[81]. All of these studies point to the central significance of the G3 domain: it facilitates GAG chain attachment and enhances product secretion. Recently, we have designed experiments to dissect the roles of aggrecan G3 domain in GAG modification and product secretion. Our studies demonstrated that the cysteine residues in the CRD motif and the CBP motif affect product secretion[82]. The functions of the G3 domain in GAG modification and product secretion are separable: GAG modification overrides its effect on product secretion but not vice versa.

It should be pointed out that the G3 domain of versican might mediate other cell activities. For example, versican G3 domain enhances cell proliferation and inhibits cell differentiation[19], [20]. The two EGF-like repeats situated in the G3 domain are critical for this effect. Their deletion inhibits proliferation of astrocytoma cells[83]. Recently, we have demonstrated that versican G3 domain binds to b1-introgrin and modulates cell adhesion and apoptosis[84].However, these functions have not been detected in aggrecan G3 domain.

\section{Conclusion}

Cartilage is a unique tissue whose properties are the result of a complex interaction of many types of molecules. Chief among these is aggrecan, a multifaceted proteoglycan whose multiple domains confer upon it diverse vital roles in stabilizing the ECM and forming the hydrated pressure- resistant gel that lubricates our joints. Understanding the structure and functions of aggrecan and other ECM molecules will be key to altering the natural history of degenerative joint disease.

\section{REFERENCES}

[1] Hardingham TE, Fosang AJ. The structure of aggrecan and its turnover in cartilage. J Rheumatol Suppl 1995; 43: 86-90.

[2] Mansson B, Carey D, Alini M, Ionescu M, Rosenberg LC, Poole AR, Heinegard D, Saxne T. Cartilage and bone metabolism in rheumatoid arthritis. Differences between rapid and slow progression of disease identified by serum markers of cartilage metabolism. J Clin Invest 1995; 95 (3):1071-7.

[3] Wight TN, Kinsella MG, Qwarnstrom EE. The role of proteoglycans in cell adhesion, migration and proliferation. Curr Opin Cell Biol 1992; 4(5):793-801.

[4] Sajdera SW, Hascall VC. Proteinpolysaccharide complex from bovine nasal cartilage. A comparison of low and high shear extraction procedures. J Biol Chem 1969; 244(1): 77-87.

[5] Hardingham TE, Muir H. The specific interaction of hyaluronic acid with cartillage proteoglycans. Biochim Biophys Acta 1972; 279(2):401-5. [6] Hardingham TE, Muir H. Binding of oligosaccharides of hyaluronic acid to proteoglycans. Biochem J 1973; 135(4):905-8.

[7] Heinegard D, Hascall VC. Aggregation of cartilage proteoglycans. 3. Characteristics of the proteins isolated from trypsin digests of aggregates. J Biol Chem 1974; 249(13): 4250-6.

[8] Zimmermann DR, Ruoslahti E. Multiple domains of the large fibroblast proteoglycan, versican. Embo J 1989; 8 (10):2975-81.

[9] Shinomura T, Nishida Y, Ito K, Kimata K. cDNA cloning of PG-M, a large chondroitin sulfate proteoglycan expressed during chondrogenesis in chick limb buds. Alternative spliced multiforms of PG-M and their relationships to versican. J Biol Chem 1993; 268(19):14461-9.

[10] Yamada H, Watanabe K, Shimonaka M, Yamaguchi Y. Molecular cloning of brevican, a novel brain proteoglycan of the aggrecan/versican family. J Biol Chem 1994; 269 (13):10119-26.

[11] Goldstein LA, Zhou DF, Picker LJ, Minty CN, Bargatze RF, Ding JF, Butcher EC. A human lymphocyte homing receptor, the hermes antigen, is related to cartilage proteoglycan core and link proteins. Cell 1989; 56(6): 1063-72.

[12] Perkins SJ, Nealis AS, Dudhia J, Hardingham TE. Immunoglobulin fold and tandem repeat structures in proteoglycan N-terminal domains and link protein. J Mol Biol 1989; 206(4):737-53.

[13] Mow VC, Zhu W, Lai WM, Hardingham TE, Hughes C, Muir H. The influence of link protein stabilization on the viscometric properties of proteoglycan aggregate solutions. Biochim Biophys Acta 1989; 992(2):201-8.

[14] Watanabe H, Gao L, Sugiyama S, Doege K, Kimata K, Yamada Y. Mouse aggrecan, a large cartilage proteoglycan: protein sequence, gene structure and pro- 
moter sequence. Biochem J 1995; 308(Pt 2):433-40.

[15] Doege KJ, Sasaki M, Kimura T, Yamada Y. Complete coding sequence and deduced primary structure of the human cartilage large aggregating proteoglycan, aggrecan. Human-specific repeats, and additional alternatively spliced forms. J Biol Chem 1991; 266(2):894-902.

[16] Yang BB, Zhang Y, Cao L, Yang BL. Aggrecan and link protein affect cell adhesion to culture plates and to type II collagen. Matrix Biol 1998; 16(9):541-61.

[17] Lee V, Cao L, Zhang Y, Kiani C, Adams ME, Yang BB. The roles of matrix molecules in mediating chondrocyte aggregation, attachment, and spreading. J Cell Biochem 2000; 79(2):322-33.

[18] Ang LC, Zhang Y, Cao L, Yang BL, Young B, Kiani C, Lee V, Allan K, Yang BB. Versican enhances locomotion of astrocytoma cells and reduces cell adhesion through its G1 domain. J Neuropathol Exp Neurol 1999; 58(6):597605.

[19] Zhang Y, Cao L, Yang BL, Yang BB. The G3 domain of versican enhances cell proliferation via epidermial growth factor-like motifs. J Biol Chem 1998; 273(33):2134251.

[20] Zhang Y, Cao L, Kiani CG, Yang BL, Yang BB. The G3 domain of versican inhibits mesenchymal chondrogenesis via the epidermal growth factor-like motifs. J Biol Chem 1998; 273(49):33054-63.

[21] Zhang Y, Wu Y, Cao L, Lee V, Chen L, Lin Z, Kiani C, Adams ME, Yang BB. Versican modulates embryonic chondrocyte morphology via the epidermal growth factor-like motifs in G3. Exp Cell Res 2001; 263(1):33-42.

[22] Grover J, Roughley PJ. The expression of functional link protein in a baculovirus system: analysis of mutants lacking the A, B and B? domains. Biochem J 1994; 300(Pt 2):317-24

[23] Watanabe H, Yamada Y, Kimata K. Roles of aggrecan, a large chondroitin sulfate proteoglycan, in cartilage structure and function. J Biochem (Tokyo) 1998; 124(4):68793.

[24] Cao L, Zhang Y, Yang BB. Expression of the G1 domain of aggrecan interferes with chondrocyte attachment and adhesion. Matrix Biol 1998; 17(5):379-92.

[25] Cao L, Yang BB. Chondrocyte apoptosis induced by aggrecan G1 domain as a result of decreased cell adhesion. Exp Cell Res 1999; 246(2):527-37.

[26] Yang BL, Zhang Y, Cao L, Yang BB. Cell adhesion and proliferation mediated through the G1 domain of versican. J Cell Biochem 1999; 72(2):210-20.

[27] Kiani C, Lee V, Cao L, Chen L, Wu Y, Zhang Y, Adams $\mathrm{ME}$, Yang BB. Roles of aggrecan domains in biosynthesis, modification by glycosaminoglycans and product secretion. Biochem J 2001; 354(Pt 1):199-207.

[28] Yang BL, Cao L, Kiani C, Lee V, Zhang Y, Adams ME, Yang BB. Tandem repeats are involved in G1 domain inhibition of versican expression and secretion and the G3 domain enhances glycosaminoglycan modification and product secretion via the complement-binding protein-like motif. J Biol Chem 2000; 275(28):21255-61.

[29] Mort JS, Buttle DJ. Cathepsin B. Int J Biochem Cell Biol 1997; 29(5): 715-20.

[30] Mort JS, Magny MC, Lee ER. Cathepsin B: an alterna- tive protease for the generation of an aggrecan 'metalloproteinase' ? cleavage neoepitope. Biochem J 1998; 335(Pt 3)(46):491-4.

[31] Paulsson M, Morgelin M, Wiedemann H, BeardmoreGray M, Dunham D, Hardingham T, Heinegard D, Timpl R, Engel J. Extended and globular protein domains in cartilage proteoglycans. Biochem J 1987; 245(3):76372.

[32] Dennis JE, Carrino DA, Schwartz NB, Caplan AI. Ultrastructural characterization of embryonic chick cartilage proteoglycan core protein and the mapping of a monoclonal antibody epitope. J Biol Chem 1990; 265(20): 12098-103.

[33] Barry FP, Neame PJ, Sasse J, Pearson D. Length variation in the keratan sulfate domain of mammalian aggrecan. Matrix Biol 1994; 14(4):323-8.

[34] Fosang AJ, Neame PJ, Last K, Hardingham TE, Murphy G, Hamilton JA. The interglobular domain of cartilage aggrecan is cleaved by PUMP, gelatinases, and cathepsin B. J Biol Chem 1992; 267(27):19470-4.

[35] Saxne T, Heinegard D. Synovial fluid analysis of two groups of proteoglycan epitopes distinguishes early and late cartilage lesions. Arthritis Rheum 1992; 35(4):38590.

[36] Morales TI, Hascall VC. Correlated metabolism of proteoglycans and hyaluronic acid in bovine cartilage organ cultures. J Biol Chem 1988; 263(8):3632-8.

[37] Little CB, Flannery CR, Hughes CE, Mort JS, Roughley PJ, Dent C, Caterson B. Aggrecanase versus matrix metalloproteinases in the catabolism of the interglobular domain of aggrecan in vitro. Biochem J 1999; 344 Pt 1:61-8.

[38] Singer, II, Kawka DW, Bayne EK, Donatelli SA, Weidner JR, Williams HR, Ayala JM, Mumford RA, Lark MW, Glant TT, et al. VDIPEN, a metalloproteinase-generated neoepitope, is induced and immunolocalized in articular cartilage during inflammatory arthritis. J Clin Invest 1995; 95(5):2178-86.

[39] Fosang AJ, Last K, Maciewicz RA. Aggrecan is degraded by matrix metalloproteinases in human arthritis. Evidence that matrix metalloproteinase and aggrecanase activities can be independent. J Clin Invest 1996; 98 (10):2292-9.

[40] Lark MW, Bayne EK, Flanagan J, Harper CF, Hoerrner LA, Hutchinson NI, Singer, II, Donatelli SA, Weidner JR, Williams HR, Mumford RA, Lohmander LS. Aggrecan degradation in human cartilage. Evidence for both matrix metalloproteinase and aggrecanase activity in normal, osteoarthritic, and rheumatoid joints. J Clin Invest 1997; 100(1):93-106.

[41] Arner EC. Effect of animal age and chronicity of interleukin-1 exposure on cartilage proteoglycan depletion in vivo. J Orthop Res 1994; 12(3):321-30.

[42] Lewthwaite J, Blake SM, Hardingham TE, Warden PJ, Henderson B. The effect of recombinant human interleukin 1 receptor antagonist on the induction phase of antigen induced arthritis in the rabbit. J Rheumatol 1994; 21(3):467-72.

[43] Cawston T. Blocking cartilage destruction with metalloproteinase inhibitors: a valid therapeutic target? 
Ann Rheum Dis 1993; 52(11):769-70.

[44] Buttle DJ, Handley CJ, Ilic MZ, Saklatvala J, Murata M, Barrett AJ. Inhibition of cartilage proteoglycan release by a specific inactivator of cathepsin $B$ and an inhibitor of matrix metalloproteinases. Evidence for two converging pathways of chondrocyte-mediated proteoglycan degradation. Arthritis Rheum 1993; 36(12):1709-17.

[45] Seed MP, Ismaiel S, Cheung CY, Thomson TA, Gardner CR, Atkins RM, Elson CJ. Inhibition of interleukin 1 beta induced rat and human cartilage degradation in vitro by the metalloproteinase inhibitor U27391. Ann Rheum Dis 1993; 52(1):37-43.

[46] Larsson E, Mussener A, Heinegard D, Klareskog L, Saxne $\mathrm{T}$. Increased serum levels of cartilage oligomeric matrix protein and bone sialoprotein in rats with collagen arthritis. Br J Rheumatol 1997; 36(12):1258-61.

[47] Sandy JD, Flannery CR, Neame PJ, Lohmander LS. The structure of aggrecan fragments in human synovial fluid. Evidence for the involvement in osteoarthritis of a novel proteinase which cleaves the Glu 373-Ala 374 bond of the interglobular domain. J Clin Invest 1992; 89(5): 1512-6.

[48] Tortorella MD, Burn TC, Pratta MA, Abbaszade I, Hollis JM, Liu R, Rosenfeld SA, Copeland RA, Decicco CP, Wynn R, Rockwell A, Yang F, Duke JL, Solomon K, George H, Bruckner R, Nagase H, Itoh Y, Ellis DM, Ross H, Wiswall BH, Murphy K, Hillman MC, Jr., Hollis GF, Arner EC, et al. Purification and cloning of aggrecanase-1: a member of the ADAMTS family of proteins. Science 1999; 284 (5420):1664-6.

[49] Lark MW, Williams H, Hoernner LA, Weidner J, Ayala JM, Harper CF, Christen A, Olszewski J, Konteatis Z, Webber R, et al. Quantification of a matrix metalloproteinase-generated aggrecan G1 fragment using monospecific anti-peptide serum. Biochem J 1995; 307(Pt 1)(4):245-52.

[50] Lohmander LS, Hoerrner LA, Lark MW. Metalloproteinases, tissue inhibitor, and proteoglycan fragments in knee synovial fluid in human osteoarthritis. Arthritis Rheum 1993; 36(2):181-9.

[51] Hughes CE, Caterson B, Fosang AJ, Roughley PJ, Mort JS. Monoclonal antibodies that specifically recognize neoepitope sequences generated by 'aggrecanase' and matrix metalloproteinase cleavage of aggrecan: application to catabolism in situ and in vitro. Biochem J 1995; 305(Pt 3):799-804.

[52] Lark MW, Gordy JT, Weidner JR, Ayala J, Kimura JH, Williams HR, Mumford RA, Flannery CR, Carlson SS, Iwata M, et al. Cell-mediated catabolism of aggrecan. Evidence that cleavage at the And quot;aggrecanase and quot; site (Glu373-Ala374) is a primary event in proteolysis of the interglobular domain. J Biol Chem 1995; 270(6):2550-6.

[53] Poole AR, Ionescu M, Swan A, Dieppe PA. Changes in cartilage metabolism in arthritis are reflected by altered serum and synovial fluid levels of the cartilage proteoglycan aggrecan. Implications for pathogenesis. J Clin Invest 1994; 94(1):25-33.

[54] Poole AR. Immunochemical markers of joint inflammation, skeletal damage and repair: where are we now? Ann Rheum Dis 1994; 53(1):3-5.

[55] Ratcliffe A, Doherty M, Maini RN, Hardingham TE. Increased concentrations of proteoglycan components in the synovial fluids of patients with acute but not chronic joint disease. Ann Rheum Dis 1988; 47(10):826-32.

[56] Fosang AJ, Hardingham TE. Isolation of the N-terminal globular protein domains from cartilage proteoglycans. Identification of G2 domain and its lack of interaction with hyaluronate and link protein. Biochem J 1989; 261 (3):801-9.

[57] Watanabe H, Cheung SC, Itano N, Kimata K, Yamada Y. Identification of hyaluronan-binding domains of aggrecan. J Biol Chem 1997; 272(44):28057-65.

[58] Fosang AJ, Hardingham TE. 1-C-6 epitope in cartilage proteoglycan G2 domain is masked by keratan sulphate. Biochem J 1991; 273(Pt 2):369-73.

[59] Antonsson P, Heinegard D, Oldberg A. The keratan sulfate-enriched region of bovine cartilage proteoglycan consists of a consecutively repeated hexapeptide motif. J Biol Chem 1989; 264(27):16170-3.

[60] Hopwood JJ, Robinson HC. The alkali-labile linkage between keratan sulphate and protein. Biochem J 1974; 141(1):57-69.

[61] Hascall VC, Riolo RL. Characteristics of the proteinkeratan sulfate core and of keratan sulfate prepared from bovine nasal cartilage proteoglycan. J Biol Chem 1972; 247(14):4529-38.

[62] Nieduszynski IA, Huckerby TN, Dickenson JM, Brown GM, Tai GH, Morris HG, Eady S. There are two major types of skeletal keratan sulphates. Biochem J 1990; 271(1):243-5.

[63] Krueger RC, Jr., Fields TA, Hildreth Jt, Schwartz NB. Chick cartilage chondroitin sulfate proteoglycan core protein. I. Generation and characterization of peptides and specificity for glycosaminoglycan attachment. J Biol Chem 1990; 265(20):12075-87.

[64] McNicol D, Roughley PJ. Extraction and characterization of proteoglycan from human meniscus. Biochem $J$ 1980; 185(3):705-13.

[65] Bourdon MA, Krusius T, Campbell S, Schwartz NB, Ruoslahti E. Identification and synthesis of a recognition signal for the attachment of glycosaminoglycans to proteins. Proc Natl Acad Sci USA 1987; 84(10):3194-8.

[66] Brennan MJ, Oldberg A, Pierschbacher MD, Ruoslahti E. Chondroitin/dermatan sulfate proteoglycan in human fetal membranes. Demonstration of an antigenically similar proteoglycan in fibroblasts. J Biol Chem 1984; 259 (22):13742-50.

[67] Chopra RK, Pearson CH, Pringle GA, Fackre DS, Scott PG. Dermatan sulphate is located on serine- 4 of bovine skin proteodermatan sulphate. Demonstration that most molecules possess only one glycosaminoglycan chain and comparison of amino acid sequences around glycosylation sites in different proteoglycans. Biochem J 1985; 232 (1):277-9.

[68] Oldberg A, Hayman EG, Ruoslahti E. Isolation of a chondroitin sulfate proteoglycan from a rat yolk sac tumor and immunochemical demonstration of its cell surface localization. J Biol Chem 1981; 256(21):10847-52. 
[69] Bourdon MA, Oldberg A, Pierschbacher M, Ruoslahti E. Molecular cloning and sequence analysis of a chondroitin sulfate proteoglycan cDNA. Proc Natl Acad Sci USA 1985; 82(5): 1321-5.

[70] Sant AJ, Cullen SE, Giacoletto KS, Schwartz BD. Invariant chain is the core protein of the Ia-associated chondroitin sulfate proteoglycan. J Exp Med 1985; 162(6): 1916-34.

[71] Upholt WB, Chandrasekaran L, Tanzer ML. Molecular cloning and analysis of the protein modules of aggrecans. Exs 1994; 70(4):37-52.

[72] McCormick D, van der Rest M, Goodship J, Lozano G, Ninomiya Y, Olsen BR. Structure of the glycosaminoglycan domain in the type IX collagen-proteoglycan. Proc Natl Acad Sci USA 1987; 84(12):4044-8.

[73] Fulop C, Walcz E, Valyon M, Glant TT. Expression of alternatively spliced epidermal growth factor-like domains in aggrecans of different species. Evidence for a novel module. J Biol Chem 1993; 268(23):17377-83.

[74] Halberg DF, Proulx G, Doege K, Yamada Y, Drickamer $\mathrm{K}$. A segment of the cartilage proteoglycan core protein has lectin-like activity. J Biol Chem 1988; 263(19):948690.

[75] Ujita M, Shinomura T, Ito K, Kitagawa Y, Kimata K. Expression and binding activity of the carboxyl-terminal portion of the core protein of PG-M, a large chondroitin sulfate proteoglycan. J Biol Chem 1994; 269(44): 27603-9.

[76] Aspberg A, Binkert C, Ruoslahti E. The versican C-type lectin domain recognizes the adhesion protein tenascinR. Proc Natl Acad Sci USA 1995; 92(23):10590-4.

[77] Aspberg A, Miura R, Bourdoulous S, Shimonaka M, Heinegard D, Schachner M, Ruoslahti E, Yamaguchi Y. The C-type lectin domains of lecticans, a family of aggregating chondroitin sulfate proteoglycans, bind tenascin$\mathrm{R}$ by protein-protein interactions independent of carbohydrate moiety. Proc Natl Acad Sci USA 1997; 94(19):
10116-21.

[78] Li H, Schwartz NB, Vertel BM. cDNA cloning of chick cartilage chondroitin sulfate (aggrecan) core protein and identification of a stop codon in the aggrecan gene associated with the chondrodystrophy, nanomelia. J Biol Chem 1993; 268(31):23504-11.

[79] Watanabe H, Kimata K, Line S, Strong D, Gao LY, Kozak CA, Yamada Y. Mouse cartilage matrix deficiency (cmd) caused by a 7 bp deletion in the aggrecan gene. Nat Genet 1994; 7(2):154-7.

[80] Kimata K, Barrach HJ, Brown KS, Pennypacker JP. Absence of proteoglycan core protein in cartilage from the $\mathrm{cmd} / \mathrm{cmd}$ (cartilage matrix deficiency) mouse. J Biol Chem 1981; 256(13):6961-8.

[81] Domowicz MS, Pirok EW, 3rd, Novak TE, Schwartz NB. Role of the C-terminal G3 domain in sorting and secretion of aggrecan core protein and ubiquitin-mediated degradation of accumulated mutant precursors. J Biol Chem 2000; 275(45):35098-105.

[82] Chen L, Wu Y, Lee V, Kiani C, Adams ME, Yao Y, Yang BB. The folded modules of aggrecan G3 domain exert two separable functions in glycosaminoglycan modification and product secretion. J Biol Chem 2002; 277(4): 2657-65.

[83] Wu Y, Zhang Y, Cao L, Chen L, Lee V, Zheng PS, Kiani C, Adams ME, Ang LC, Paiwand F, Yang BB. Identification of the motif in versican G3 domain that plays a dominant-negative effect on astrocytoma cell proliferation through inhibiting versican secretion and binding. J Biol Chem 2001; 276(17):14178-86.

[84] Wu Y, Chen L, Zhang PS, Yang BB. b1-Integrin mediated glioma cell adhesion and free radical-induced apoptosis are regulated by binding to a C-terminal domain of PGM/versican. J Biol chem 2001; 277 JBC paper in press, published on January 22, 2002 as Manuscript M110748200. 